\title{
Composite augmentation phalloplasty: personal experience after 275 patients
}

\author{
Juan Monreal \\ Consultant Plastic Surgeon, Hospital Moncloa, Avenida de Valladolid, 83, 28008 Madrid, Spain. \\ Address for correspondence: Dr. Juan Monreal, Londres, 54-1D, Torrejon de Ardoz, 28850 Madrid, Spain. E-mail: juanmonreal@gmail.com
}

\begin{abstract}
Aim: To report the author's experience in augmentation phalloplasty by studying a retrospective series of patients who underwent fat grafting for girth enhancement or a composite technique based on suspensory ligament release plus fat grafting performed simultaneously. Methods: The author analyzed retrospectively the outcomes of 275 augmentation phalloplasty procedures performed in 259 patients until November 2013. Of these, 127 correspond to girth augmentation with fat grafting and 148 to composite augmentation phalloplasty (girth augmentation with fat grafting and length improvement by suspensory ligament release). In 16 patients girth and length enhancement were performed in two separate procedures. Results: Of this 259 patients, 87 underwent postoperative follow-up for at least 12 months and 160 patients underwent follow-up for at least 6 months. The average increase in circumference at 6 months was $1.7 \mathrm{~cm}(1.57 \mathrm{~cm}$ at 12 months $)$ and the average increase in length of $3.2 \mathrm{~cm}$ (3.1 cm at 12 months). Twenty-two patients showed minor complications that were treated without sequelae and without influencing the final result. Conclusion: By judicious use of currently available techniques, it is possible to achieve stable increases in penis size. The use of composite techniques provides better final results than the use of individual techniques performed alone due to the increase of the actual volume of the penis. An adequate informed consent is essential in all patients due to the unrealistic expectations expressed by the majority of them.
\end{abstract}

Key words:

Adipose tissue, fat grafting, lipofilling, penis augmentation

\section{INTRODUCTION}

One of the traditional benefits that aesthetic surgery offers to patients is the improvement in self-esteem. The appearance of the external genitalia, both male and female, can have similar degrees of influence on social relationships and may cause a concern similar to that generated by a lipodystrophy or a nose with inadequate proportions. Given the unrealistic expectations (sometimes fanciful) that these patients tend to assume about the anticipated results, it is essential to properly and thoroughly inform patients about treatment characteristics,

\begin{tabular}{|l|l|}
\multicolumn{2}{|c|}{ Access this article online } \\
\hline Quick Response Code: & Website: \\
\hline & www.parjournal.net \\
\hline & \\
\hline
\end{tabular}

limitations, and what results they can expect. Although a large number of patients who are interested in genital aesthetic surgery request a consult for purely aesthetic motivation, as in other domains of aesthetic surgery, there are many in which a functional defect coexists with the aesthetic burden that can worsen patient experience in sexual or social relationships.

Generally speaking, aesthetic surgery of male genitalia is focused on increasing penis measures in both length and girth. There are, however, ancillary techniques that work to improve the "visual" size of the penis and scrotum. At the present time, there is only one technique capable of increasing girth in erection but none (at least known to the author) capable of increasing dynamically the length in erection. Therefore, currently, existing techniques are able to increase the girth in erection or flaccidity but not both. There are surgical techniques that increase the length in the flaccid state, but no reports known to the author that describe increasing the length of the penis in erection. For these reasons the enhancement of the penis size is better achieved by performing several surgical techniques 
simultaneously or in stages. If it is assumed that the penis is a cylindrical body, a more successful outcome should seek to increase the entire volume (diameter and length) than only one of the dimensions [Figure 1]. There is only one exception to this approach which is the treatment of a buried or partially buried penis. In this case, given the importance of adequate pubic skin redraping and marking the new peneopubic angle, the author recommends girth enhancement in a second staged step.

An important factor that is difficult to standardize is the measurement of penile length and girth in flaccidity and erection. Several ways to do this are postulated, with the main objective of obtaining an accurate estimate of the increase in these measures in postoperative follow-up for comparative purposes. As a general rule the established mean normal values of flaccid length are between $7.2 \mathrm{~cm}$ and $11.0 \mathrm{~cm}$ measured from the peneopubic angle to the tip of the glans, with an average of $9.5 \mathrm{~cm}$. A normal girth will vary between $7.7 \mathrm{~cm}$ and $12.0 \mathrm{~cm}$, with an average of $9.56 \mathrm{~cm} .^{[1]}$ It is extremely important that the surgeon standardize the method for taking these measurements to maintain consistency in daily practice.

The author presents his experience in a retrospective study of 275 augmentation phalloplasty procedures. The final analysis focuses on the main increase in penis measurements (girth and length) in the flaccid state and the stability of improvement over time.

\section{METHODS}

The author analyzed retrospectively the outcomes of 275 augmentation phalloplasty procedures performed in 259 patients. The main motivation for all patients was to achieve greater penis size without the development of any form of impotence or sexual dysfunction. Mean preoperative penis dimensions in the flaccid state were $8.9 \mathrm{~cm}$ in girth (range, 6.5-10.2 cm) and $9.2 \mathrm{~cm}$ in length (range, $7.4-12.2 \mathrm{~cm}$ ). Of the 275 procedures, 127 underwent girth enhancements with fat grafting and 148 were undertaken for composite phalloplasties (girth enhancement with fat grafting and length improvement by suspensory ligament release). In 16 patients, these procedures were performed in two surgical stages, not $<6$ months apart. All patients

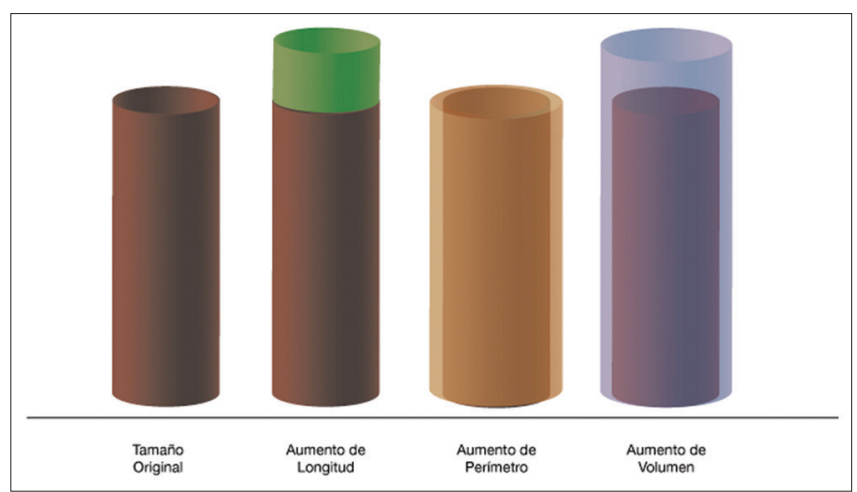

Figure 1: The penis can be approximated to a cylinder, and thus, enhancement of girth and length at the same time improves real volume. From left to right: original size, length improvement, girth improvement, both simultaneously signed the corresponding informed consent. The age of patients ranged from 23 to 57 years with a mean age of 38 years. All cases of girth enhancement with fat grafts were performed under local anesthesia with sedation on an ambulatory basis; composite phalloplasty were performed under spinal anesthesia on an ambulatory basis as well. All pre and postoperative measures and pictures were taken in forced flaccidity (applying a light traction on the penis for about $3 \mathrm{~s}$ ). Postoperative measurements were scheduled on the day after the operative procedure and again at $1,4,6$, and 12 months postoperatively.

\section{Girth enhancement with fat grafting}

The technique of fat grafting used in this series has been published previously. ${ }^{[2,3]}$ Briefly, lipoaspirate is harvested with a 20-hole $3 \mathrm{~mm}$ cannula (Quirumat, Spain). The lipoaspirate is washed with Ringer's lactate, and layer separation is obtained by decanting for about $30 \mathrm{~min}$. Once processed, the washed fat is injected under the dartos and Buck's fascia. The engrafting process must be performed with extreme caution, placing fat fragments of no $>3 \mathrm{~mm}$ in diameter to ensure a proper take and prevent necrosis and cyst formation. Whether girth enhancement is performed alone or in combination with suspensory ligament release, the infiltration cannula is advanced from the peneo-pubic angle towards the preputial skin or circumcision scar. The fat is injected in a retrograde fashion and distributed all around the girth; this is tailored to the needs of each patient, from the peneo-pubic angle to the coronal sulcus scar (if the patient is circumcised) or to the foreskin proper (if he is not circumcised). The distribution of fat is particularly important in the penis foreskin to prevent unaesthetic nodules or bulges or the presence of an offset devoid of fat when the patient removes the foreskin. This technique can be performed alone $(127+16$ cases in our series) or preferably at the same time as the suspensory ligament release (148 patients in our series).

The behavior of fat grafted to the penis is mostly the same as when performed in other body areas. Although postoperative swelling usually takes about 6 weeks to disappear, the volume loss in the grafts should stabilize by $3-4$ months; at this timepoint the improvement in girth must be stable.

\section{Composite augmentation phalloplasty}

Figures 2 and 3 summarize the sequence of steps performed during suspensory ligament release as done in

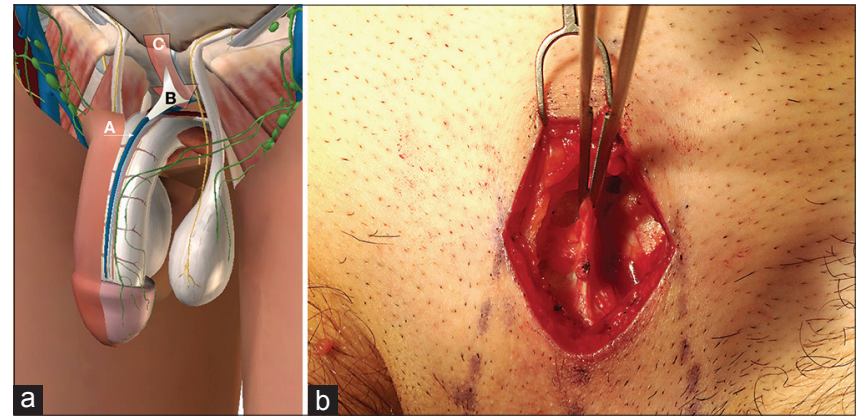

Figure 2: (a) Anatomy of the suspensory ligament: (A) deep neurovascular bundle; (B) suspensory ligament; (C) surgical approach. (b) Pick-ups are shown grasping the most caudal and superficial aspect of the ligament 
the current series. Although composite phalloplasty can be performed under local anesthesia and sedation, the author prefers spinal anesthesia that adds little morbidity and enhances patient comfort. The procedure begins with a $3.5 \mathrm{~cm}$ incision located about $2.5-3.0 \mathrm{~cm}$ cranial to the peneo-pubic angle. The first step is to perform the dissection and release of the fascial and fasciocutaneous attachments. The dissection then proceeds down to the front edge of the suspensory ligament. Thus, the release must be performed directly from the attachments to the symphyseal ligament to avoid accidental damage to deep penile neurovascular structures. The release is then carried further down, stopping at the start of the pelvic floor. The author usually does not release bone attachments except in cases of micropenis. After the ligament release is complete, corpora cavernosa will move easily forward and downward, creating a dead space between these structures and the pubic bone; This dead space must be filled with local tissues; the availability of these tissues can be extremely variable depending on the body mass index of the patient. In slim patients it is usually necessary to take the fat that surrounds the spermatic cords. When there is enough pubic fat, adipofascial flaps can be tailored and turned down as described by Hinderer and Espinosa. ${ }^{[4]}$ Available tissues are interposed inside the dead space created by the ligament release while simultaneously pulling on the penis and checking on the stability of the repair.

Upon completion of these steps, a skin gap can be observed and that is caused by penis advancement. Although a variety

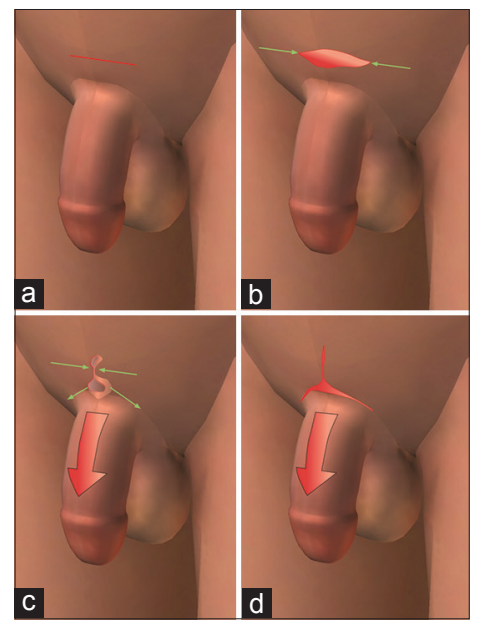

Figure 3: Sequence of suspensory ligament release as performed by the author. (a) Transverse incision; (b) symphysis approach and complete release; (c) transverse closure, advancement and dog ears; (d) dog ear treatment

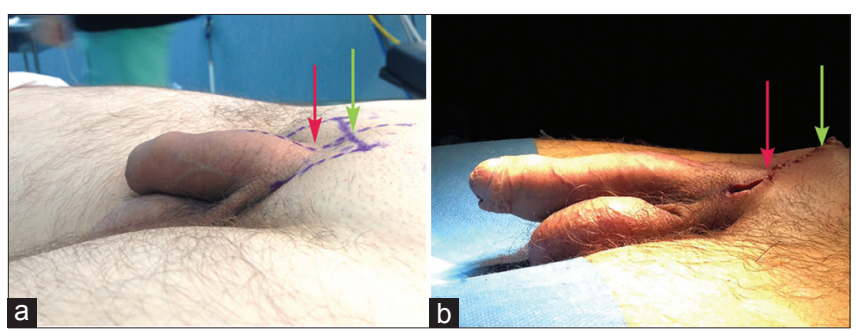

Figure 5: Intraoperative views (a) before and (b) after completion of composite augmentation phalloplasty. Green arrows depict initial incision location. Red arrows mark peneopubic angle of skin plasties can be planned in advance and performed at the beginning of the procedure, the author prefers to do this once the penis advancement has been completed, to modify for each situation. Treatment of the skin gap begins by closing the defect in a horizontal fashion [Figure 4]. This closure produces two dog ears that will provide the final measure of skin advancement. The distal dog ear is tailored to provide a $\mathrm{Y}$ or $\mathrm{T}$ advancement. The proximal dog ear is usually smaller and can be managed by defatting and direct closure; in about 2-3 months it will flat tenon on its own. Performed correctly, closure of the skin by an advancement plasty stabilizes and maintains the improvement in length [Figure 5]. It must be kept in mind that an overly ambitious cutaneous advancement usually results in the incorporation of hairy skin and some scrotalization of the penis shaft which worsens the aesthetic result. Before epidermal closure, the author inserts a vacuum drain and then proceeds to girth augmentation with fat grafting as previously described. All sutures used including epidermal closure can be performed with $4 / 0$ absorbable monofilament.

As a rule composite augmentation phalloplasty can be performed on an outpatient basis. The drain is removed after $24 \mathrm{~h}$ and antibiotics are continued for 3 days. After

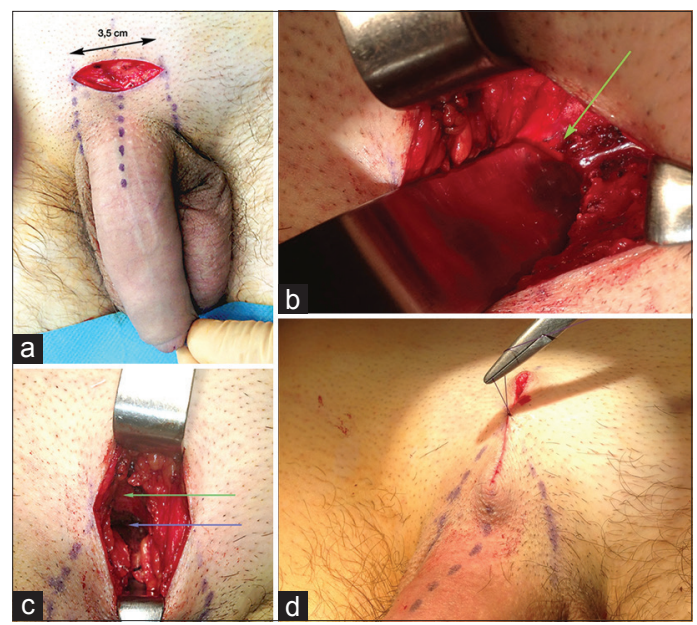

Figure 4: Sequence of suspensory ligament release as performed by the author. (a) Transverse incision; (b) symphysis approach; (c) complete release (green arrow: pubis, blue arrow: urogenital diaphragm); (d) transverse initial closure

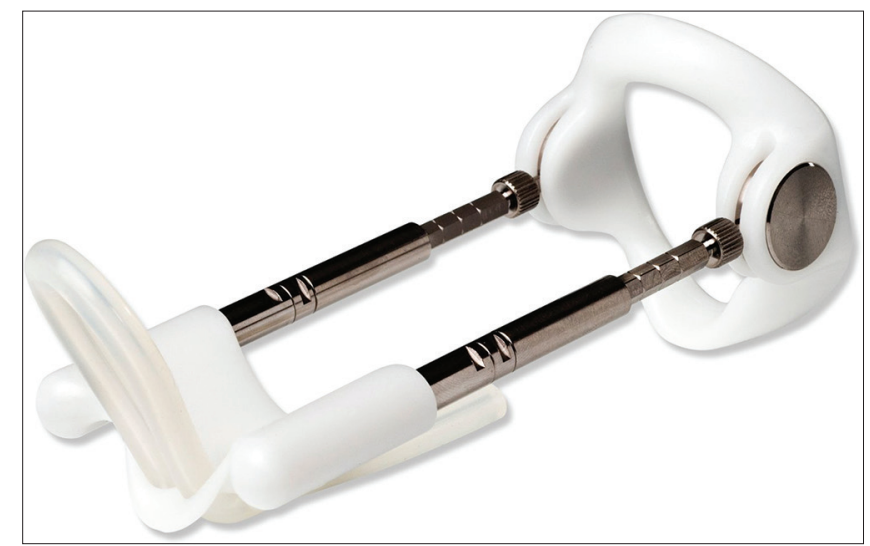

Figure 6: Original model of traction system (JES extender). Today all brands look the same as the original 
15 days, patients can start using the traction system if previously circumcised. In noncircumcised patients traction must be delayed until foreskin swelling has disappeared. The author recommends the use of an extender [Figure 6] because it ensures control over initial scar maturation and helps to prevent ligament reattachment. In addition, when used correctly, additional length is added to that offered by the surgical procedure. All patients are encouraged to maintain sexual abstinence during the 1 st month postoperatively.

\section{RESULTS}

Of the 259 patients who underwent surgery, 160 provided a 6 months follow-up and 87 completed 12 months of follow-up. In 99 patients follow-up was $<6$ months. The average increase in girth was $1.7 \mathrm{~cm}$ at 6 months and $1.6 \mathrm{~cm}$ at 12 months and the mean increase in length was $3.1 \mathrm{~cm}$ and $3.2 \mathrm{~cm}$ at 6 and 12 months, respectively. In 22 patients ( $8 \%$ of the series) the author detected minor complications that were treated without sequelae and without influencing the final result. No patient reported functional problems or difficulty in sexual activity after the second postoperative month. Postoperative length gain did not change during the first 6 months of follow-up. Patients who used the extender for at least 3 months after surgery achieved modest additional increases in length, which did not exceed $1.3 \mathrm{~cm}$. The author was not able to properly analyze the increase in erection measurements due to lack of data. Figures 7-9 represent average results of composite augmentation phalloplasty. Figures 10 and 11 represent average results of penis girth enhancement with fat grafting.

Minor complications encountered after phalloplasty were combined infection: marginal wound dehiscence (3 cases, $2 \%$ ), the development of small seromas that required aspiration (5 cases, 3.4\%, especially when performing the suprapubic adipofascial flap), liponecrotic cysts that were resectable secondarily (4 cases, $2.7 \%$ in the first 4 years of experience). There were no incidents of keloid scar formation, however, in 5 cases the final scar was considered hypertrophic. The author currently recommends placement of silicone sheets or gels as part of the postoperative care. The use of a postoperative traction system is not mandatory but helps to minimize the chances of abnormal scarring and to gain extra length. Lack of compliance with the extender device or the presence of erosion caused by the pulling ring is a common cause for abandoning the use of postoperative traction. The author did not encounter any cases of postoperative paradoxical shortening.

Regarding girth enhancement performed as a stand-alone procedure, the complications were liponecrotic cysts in $7 \%$ of patients ( 9 cases in the first 5 years of experience), 1 case of postoperative infection that needed a complete antibiotic course $(0.8 \%)$, and 1 case of fat overgrowth due to extreme weight gain (0.8\%) [Figure 12]. Lack of abstinence, especially during the first 2 weeks, can certainly cause the loss of grafted fat to some degree, so the patient should be warned about this.

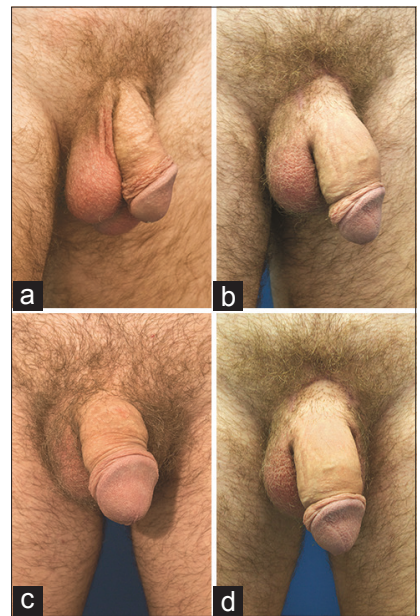

Figure 7: Case 1. preoperative and 11 months postoperative views of composite augmentation phalloplasty ( $40 \mathrm{~mL}$ of fat). (a and c) Preoperative; (b and d) 11 months postoperative

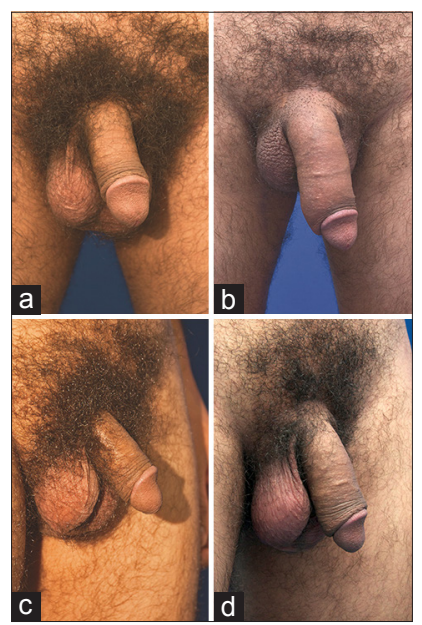

Figure 8: Case 2. preoperative and 16 months postoperative views of composite augmentation phalloplasty ( $55 \mathrm{~mL}$ of fat). (a and c) Preoperative; (b and d) 16 months postoperative

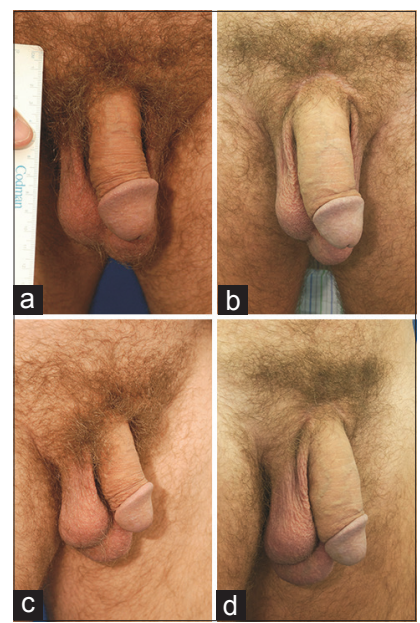

Figure 9: Case 3. preoperative and 12 months postoperative views of composite augmentation phalloplasty (65 mL of fat). (a and c) Preoperative; (b and d) 12 months postoperative

\section{DISCUSSION}

Standard measurement of the penis has been a controversial issue and a subject of discussion 


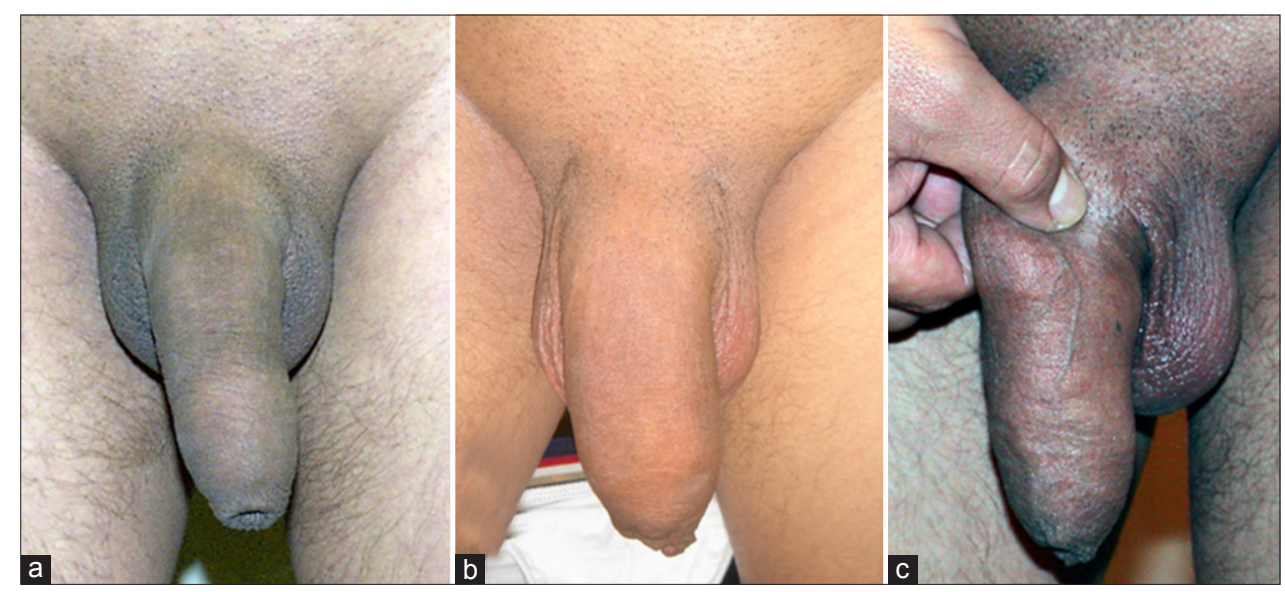

Figure 10: Case 4. preoperative and 3 years postoperative view of girth enhancement with fat grafting in two sessions (30 +40 mL). (a) Preoperative; (b) 3 years postoperative; (c) pinch of implanted fat

for a long time. The racial controversy introduces more variables, which can influence decisions about justifying augmentation phalloplasty. ${ }^{[5,6]}$ The enhancement of penis size can be achieved using surgical or nonsurgical procedures. Unfortunately the uses of unproven techniques or synthetic fillers have made these treatments notorious for their sequelae or bad results. ${ }^{[7]}$ Nonsurgical techniques that use traction by weights have been employed by many cultures over the centuries and are based on cultural, religious or aesthetic purposes. ${ }^{[8,9]}$ The modern age of these treatments began at the end of the 1990's with the JES Extender device. These techniques exploit the ability of tissues to respond to physical stimuli as traction or expansion with hyperplasia and cell division, a well-known behavior used by plastic surgeons worldwide.

As in any medical or surgical procedure using expansion or distraction, while using a penis extender a pulling force of a certain intensity must be applied and must be as continuous as possible and for a minimum time period and hence that the biological phenomena responsible for tissue modification are started. The use of these devices often requires a great deal of diligences on the part of patients to get results that are minimally satisfactory. Erosions are sometimes produced by traction ring or by irregular use of the device and are some of the factors that negatively affect the results, which may be minimal. In any case, an adequate knowledge about the management of these devices must be present in the armamentarium of the surgeon performing phalloplasties, since it can be an extremely useful complement to the surgical procedure to secure, maintain and/or improve the outcome. The author always uses traction as an adjunct to surgical treatment and never as a stand-alone treatment.

As in the case of surgical lengthening, there is not a single surgical solution to increase the thickness or perimeter of the penis. ${ }^{[10,11]}$ Pericavernous techniques provide girth augmentation in flaccid penises but little or no improvement in erection. These techniques try to obtain an increase in girth by implanting some of the

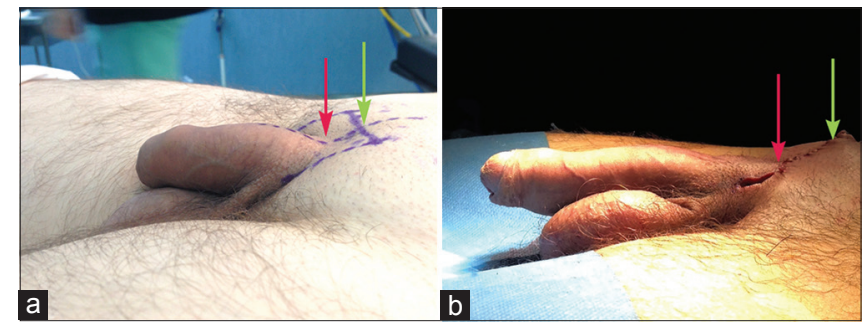

Figure 11: Case 5. preoperative and 9 months postoperative view of girth enhancement with fat grafting (35 mL of fat). (a) Preoperative; (b) 9 months postoperative

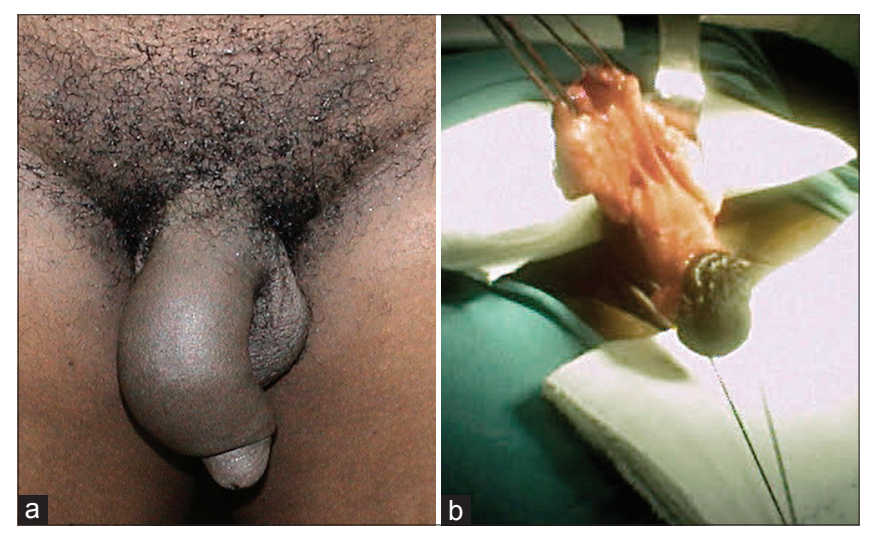

Figure 12: (a) Preoperative view of fat graft overgrowth due to $26 \mathrm{~kg}$ of weight gain 4 years after girth augmentation; (b) intraoperative view of complete lipectomy

available grafts (fat or dermofat grafts) or biological implants (acellullar dermis) around the penis shaft outside the albuginea and under the dartos fascia. More advanced techniques using resorbable matrices together with autologous fibroblasts have also been described with good results. ${ }^{[12,13]}$ Albugineal techniques get good results in erection but none in flaccidity. ${ }^{[14]}$ The injection of synthetic fillers is probably one of the options that are frequently performed, but may cause many deformities and complications. All of these techniques are more invasive and thus can yield a greater number and severity of complications. Fat grafting is probably the least aggressive technique but requires fine control and technique to avoid complications and poor results. It was introduced as a technique for girth enhancement 
in the mid-1980's and continues to be used due of its versatility.

Traction treatments are the only ones able to achieve effective length improvements of the penis, producing an increase in actual tissue mass and at the same time "softening" the corpora cavernosa fascial and osseous attachments. The combined use of both types of treatments (suspensory ligament release and extender use) can produce the best outcome by extending the "hidden" part of the penis with generation of additional tissue. All penis lengthening techniques are based on the release of natural physiologic anchors that bind the corpora cavernosa to the pubic symphysis, isquiopubic bone branches, and abdominal aponeurotic expansions. Although this lengthening can be performed through a simple transverse incision or even using an endoscope (not published), a number of ancillary skin advancements can be performed to ease ligament release and to procure advancement and stabilization, and thus prevent length loss due to scar contracture. In this regard several techniques have been published such as M-plasty (popularized in China and reported by Reed ${ }^{[15)}$ ), VY advancement (Roos and Lissoos ${ }^{[16]}$ ), Z-plasty (Horton ${ }^{[17]}$ ), double Z-plasty (Alter ${ }^{[18,19]}$ ) and personal techniques of Abecassis ${ }^{[20]}$ and Panfilov. ${ }^{[21]}$ Although the author supports the use of local tissues to fill the advancement gap to additionally maintain advancement, some authors have published their experience inserting small testicular prostheses as spacers with no better results. ${ }^{[22]}$

In our practice performing suspensory ligament release, skin flap advancement together with simultaneous girth enlargement using fat grafts is the most satisfactory approach to increase penis size. This composite technique generates real volume increase which results in a better perceptual outcome for the patient. Additionally, the increase in shaft convexity and downward position creates the illusion of a longer penis as well. The incidence of complications or side effects is relatively low and easy to solve without affecting final results. A thorough knowledge of regional anatomy helps to prevent serious or minor complications. There are two good safety rules: perform the ligament release as close to the bone as possible and do not perform any grafting that may exceed the capacity of the tissue. Although liponecrotic cyst occurrence can be solved easily, damage to the deep penile neurovascular structures usually has disastrous consequences. Patient satisfaction after augmentation phalloplasty is lower when compared with other popular aesthetic surgical procedures. In our series, only $32 \%$ perceived the result as very good and an additional $43 \%$ as good, in spite of being clearly informed about the limitations and outcomes for other patients and follow-up demonstration of improved average measurements. As with any other aesthetic surgery procedure, informing the patient is extremely important in achieving an adequate degree of satisfaction. Two relatively distinct patient groups exists: those who present with a real hypoplasia and those cases of body dismorphic disorder. The former tend to show higher degrees of satisfaction and understand easily the limitations of the techniques. Patients with unrealistic expectations that do not understand this information must be discouraged from the procedure because the level of disappointment will likely be very high. It is extremely important not to assure the patient that any type of result will be an improvement; patients should understand that it is possible to see no improvement, an event caused usually by an inadequate scar contraction.

Composite augmentation phalloplasty techniques are safe and reproducible and yield satisfactory results if properly performed. Although apparently easy to perform, a thorough knowledge of anatomy and grafting techniques is needed to get good results and avoid complications. Volumetric enhancement by ligament release and cutaneous advancement together with an increase in girth with fat grafting is probably a safer option, with better results and lower morbidity. The future of penis enlargement will be enhanced with techniques that provide dynamic improvements in size, possibly through the use of tissue engineering.

\section{REFERENCES}

I. Mondaini N, Ponchietti R, Gontero P, Muir GH, Natali A, Caldarera E, Di Loro F, Biscioni S, Rizzo M. Penile length is normal in most men seeking penile lengthening procedures. Int J Impot Res 2002; 14:283-6.

2. Monreal J. Fat tissue as a permanent implant:new instruments and refinements. Aesthet Surg J 2003;23:213-6.

3. Monreal J. Male and female genital aesthetic surgery: basic techniques and concepts. AECEPJ 2006; I:8-17.

4. Hinderer UT, Espinosa JF. New enlargement technique with volume enhancement in penis hypoplasia and hypospadias. Cir Plast Iberlatinamer 1997;23:151-60.

5. Dillon BE, Chama NB, Honig SC. Penile size and penile enlargement surgery: a review. Int J Impot Res 2008;20:5 I 9-29.

6. Ghanem H, Glina S, Assalian P, Buvat J. Position paper: management of men complaining of a small penis despite an actually normal size. J Sex Med 2013; 10:294-303.

7. Parodi PC, Dominici M, Moro U. Penis invalidating cicatricial outcomes in an enlargement phalloplasty case with polyacrylamide gel (Formacryl). Int J Impot Res 2006; |8:3|8-2|

8. Oderda M, Gontero P. Non-invasive methods of penile lengthening: fact or fiction? BJU Int 201 I; 107:1278-82.

9. Colpi GM, Martini P, Scroppo FI, Macini M, Castiglioni F. Efficacy of daily penis-stretching technique to elongate the "small penis". Int J Impot Res 2002; | $4: 155$.

10. Shaeer O, Shaeer K. Penile girth augmentation using flaps "Shaeer's augmentation phalloplasty”: a case report. J Sex Med 2006;3:I64-9.

II. Alei G, Letizia P, Ricottilli F, Simone P, Alei L, Massoni F, Ricci S. Original technique for penile girth augmentation through porcine dermal acellular grafts: results in a 69-patient series. J Sex Med 2012;9:1945-53.

12. Jin Z,WuYG,Yuan YM, Peng J, GongYQ, Li GY, SongWD, CuiWS, He XY, Xin ZC. Tissue engineering penoplasty with biodegradable scaffold Maxpol-T cografted autologous fibroblasts for small penis syndrome. J Androl 201 I;32:49I-5.

13. Perovic SV, Byun JS, Scheplev P, Djordjevic ML, Kim JH, Bubanj T. New perspectives of penile enhancement surgery: tissue engineering with biodegradable scaffolds. Eur Urol 2006;49:139-47.

14. Austoni E, Guarneri A, Cazzaniga A. A new technique for augmentation phalloplasty: albugineal surgery with bilateral saphenous grafts -three years of experience. Eur Urol 2002;42:245-53.

15. Reed HM. Augmentation phalloplasty with girth enhancement employing autologous fat transplantation: a preliminary report. Am J Cosmet Surg 1994; I I:85-9.

16. Roos H, Lissoos I. Penis lengthening. Int J Aesthetic Restorative Surg 1994;2:89-96.

17. Horton CE, Vorstman B, Teasley D, Winslow B. Hidden penis release: adjunctive suprapubic lipectomy. Ann Plast Surg 1987; 19:131-4.

18. Alter GJ, Salgado CJ, Chim H.Aesthetic surgery of the male genitalia. Semin Plast Surg 201 1;25:189-95. 
19. Alter GJ.Augmentation phalloplasty. Urol Clin North Am 1995;22:887-902.

20. Abecassis $M$, Berreby $S, B$ Boccara $D$. Penis enlargement surgery:lipopeneosculpture for length and girth enhancement. Ann Chir Plast Esthét 2010;55:135-42.

21. Panfilov DE. Augmentative phalloplasty. Aesthetic Plast Surg 2006;30:183-97.

22. Li CY, Kayes O, Kell PD, Christopher N, Minhas S, Ralph DJ. Penile suspensory ligament division for penile augmentation: indications and results. Eur Urol 2006;49:729-33.
How to cite this article: Monreal J. Composite augmentation phalloplasty: personal experience after 275 patients. Plast Aesthet Res 2015;2:27-33.

Source of Support: Nil, Conflict of Interest: None declared.

Received: 19-09-2014; Accepted: 23-10-2014 